\title{
Cardiac magnetic resonance imaging-derived cardiac index is associated with adverse outcomes in patients with single-ventricle Fontan circulation: a preliminary study
}

\author{
Małgorzata Kowalczyk¹, Piotr Zieliński', Magdalena Marczak², Katarzyna Kożuch', \\ Magdalena Lipczyńska', Mirosław Kowalski', Piotr Hoffman¹ \\ 1 Department of Congenital Heart Diseases, National Institute of Cardiology, Warsaw, Poland \\ 2 Magnetic Resonance Imaging Unit, Department of Radiology, National Institute of Cardiology, Warsaw, Poland
}

Correspondence to: Małgorzata Kowalczyk, MD, Department of Congenital Heart Diseases, Institute of Cardiology, ul. Alpejska 42, 04-628 Warszawa, Poland, phone: +48223434400 , email: mkowalczyk1@ikard.pl Received: August 29, 2020. Revision accepted: February 5, 2021. Published online: February 22, 2021. Kardiol Pol. 2021; 79 (3): 336-338 doi:10.33963/KP.15822 Copyright by the Author(s), 2021
Introduction Single ventricle (SV) refers to a group of heart defects with only one ventricle properly developed. The term describes a spectrum of cardiovascular malformations that are not feasible for surgical partitioning of ventricular mass. ${ }^{1}$ Fontan surgery (currently most often involving total cavopulmonary connection $[\mathrm{TCPC}])$ is a palliative procedure that connects systemic and pulmonary vascular beds in series. ${ }^{2}$ This procedure creates a "neoportal system," in which the venous return flows directly to pulmonary capillaries, bypassing the heart. An SV is the only pump that generates energy for blood flow, but its role in determining cardiac output (CO) is limited. Lack of the subpulmonary ventricle, along with a surgically created connection, leads to high central venous pressure and results in decreased CO. Patients undergoing Fontan surgery are exposed to multiorgan dysfunction (Fontan-associated liver disease, chronic kidney disease, protein-losing enteropathy, among others $)^{3,4}$ and low exercise capacity. ${ }^{5}$ Those patients also have a reduced heart rate reserve. ${ }^{6}$ We hypothesized that a cardiac index (CI) derived from cardiac magnetic resonance (CMR) imaging may prove to be an effective tool for the overall assessment of Fontan circulation function. The aim of the study was to determine the usefulness of a CMR-derived $\mathrm{CI}$ as a multiorgan dysfunction marker and an adverse-outcome predictor in patients after Fontan palliation.
Methods Thirty-four patients undergoing the Fontan procedure were retrospectively enrolled in the study. Cardiopulmonary exercise testing was performed on Treadmill Ergometer ZAN Ergo 600s (Delmar Reynolds, Irvine, California, United States). Maximal oxygen uptake $\left(\mathrm{VO}_{2} \max \right)$ was measured by a modified Bruce protocol. Biochemical data were obtained using standard assay techniques. Collected parameters included serum concentrations of total protein, aspartate and alanine transaminases, total bilirubin, iron, creatinine, thyroid-stimulating hormone, and C-reactive protein. Blood cell tests provided data on hemoglobin levels, platelet and leukocyte counts, and mean corpuscular volume. Additionally, a glomerular filtration rate was estimated. Cardiac magnetic resonance imaging was performed using a 1.5 Tesla scanner (Avanto and Avantofit, Siemens Healthineers, Erlangen, Germany) to evaluate the CO. The $\mathrm{CO}$ was calculated according to the following formula: $\mathrm{CI}=\mathrm{CO} /$ body surface area. Adverse events were identified and followed up for a mean of 4 years. Those events included arrhythmia, nonelective hospitalization, death, heart transplant, lower New York Heart Association (NYHA) functional class, and decreased $\mathrm{VO}_{2}$ max.

The study protocol was approved by an ethics committee. Patient consent was not required owing to the retrospective design of the study. 
TABLE 1 Clinical and demographic characteristics of the study patients

Characteristics

Patients $(n=34)$

\begin{tabular}{lll} 
Male sex & & $15(44)$ \\
\hline Single ventricle type & Left & $25(73)$ \\
\cline { 2 - 3 } & Right & $6(18)$ \\
\cline { 2 - 3 } & Undifferentiated & $3(9)$ \\
\hline Age at cardiac MRI, y & & $25.5(21.25-30.75)$ \\
\hline Time from the Fontan procedure, y & & $17(15.25-21)$ \\
\hline
\end{tabular}

Data are presented as number (percentage) of patients or median (interquartile range).

Abbreviations: MRI, magnetic resonance imaging

Statistical analysis Study results were analyzed using the Statistica software, version 13.3 (StatSoft, Kraków, Poland). Quantitative variables were expressed as median and interquartile range (IQR). Qualitative variables were presented as numbers and percentages. The Shapiro-Wilk test was used to assess normality. The correlation among biochemical parameters, time from surgery, and CI was assessed using the Spearman rank correlation coefficient. The Mann-Whitney test was used to identify differences in CI between the study groups (Supplementary material, Table S2). A $P$ value less than 0.05 was considered significant.

Results and discussion Patients' clinical and demographic characteristics are shown in TABLE 1. The median (IQR) CMR-derived CI was $3.37(2.99-3.87) 1 / \mathrm{min} / \mathrm{m}^{2}$ (minimum, $2.1 \mathrm{l} / \mathrm{min} / \mathrm{m}^{2}$; maximum, $6.1 \mathrm{l} / \mathrm{min} / \mathrm{m}^{2}$ ). Maximal oxygen uptake ranged from $13.16 \mathrm{ml} / \mathrm{min} / \mathrm{kg}$ to $28.37 \mathrm{ml} / \mathrm{min} / \mathrm{kg}$, with a median (IQR) of $19.46(15.48-21.48) \mathrm{ml} / \mathrm{min} / \mathrm{kg}$. During a median (IQR) follow-up of 6 years and 7 months (range, 5 years and 9 months to 7 years and 1 month), 16 events occurred including 9 arrhythmias, 5 nonelective hospitalizations, and a single death. Only supraventricular tachycardia episodes were observed in 4 patients with atrial fibrillation and 2 with atrial flutter. Indications for nonelective hospitalization included arrhythmia episodes, Fontan circulation failure, and diagnostic workup of chest pain. A single patient died 8 days after Fontan conversion to the TCPC procedure. There was no significant relationship between $\mathrm{CI}$ and parameters of organ dysfunction (serum creatinine level, glomerular filtration rate, total serum protein level, bilirubin and aminotransferase levels, serum iron level, and blood count) (Supplementary material, Table S1). There was also no correlation among age, worsening of the NYHA functional class, and $\mathrm{VO}_{2}$ max reduction with $\mathrm{CI}$ (Supplementary material, Table S2). The cardiac index was significantly higher in patients who were not hospitalized compared with those who required nonelective hospitalization (Supplementary material, Table S2). A negative correlation between the time from surgery and CI was found.

The SV preload after Fontan palliation depends mainly on pulmonary flow. ${ }^{7}$ Lack of a subpulmonary pump to generate the pressure gradient and inward flow to pulmonary arteries causes chronic preload decrease. Filling-volume deprivation leads to concentric hypertrophy, diastolic dysfunction, and, as a consequence, to decreased stroke volume. Patients after TCPC also have a limited heart rate reserve, which is probably an adjustment to abnormal cardiac filling rather than sinus node dysfunction. ${ }^{6} \mathrm{~A}$ reduction of both stroke volume and heart rate leads to decreased CO and CI. Cardiac output and $\mathrm{CI}$ are hemodynamic parameters obtained by invasive methods. Hundley et $\mathrm{al}^{8}$ demonstrated a correlation of CMR-derived $\mathrm{CO}$ and standard invasive measurements. There is some evidence on the usefulness of CMR-derived CI in healthy adults and patients with congestive heart failure, ${ }^{9}$ as well as in those after the Fontan procedure. ${ }^{10}$

Cardiac output in patients with SV is decreased by $50 \%$ to $80 \%$ of the normal value for body surface area. ${ }^{11}$ Chronically low $\mathrm{CO}$ and $\mathrm{CI}$ in the case of normal heart anatomy may lead to low arterial and perfusion pressure. We hypothesized that a decreased CI can influence organ function in patients after the Fontan procedure, as organ function deteriorates in patients with congestive heart failure. ${ }^{12}$ Mori et $a l^{13}$ investigated an association between invasively measured hemodynamic parameters (CI and signs of liver and renal dysfunction in patients after the Fontan procedure). Their findings revealed no significant association between the hemodynamic measurements and end-organ dysfunction parameters, which coincides with our findings.

Cardiac output in patients after the Fontan procedure is approximately half of that of healthy controls during exercise.$^{14}$ However, we did not find a link between $\mathrm{CI}$ and $\mathrm{VO}_{2}$ max during cardiopulmonary exercise testing. There was also no correlation with the NYHA functional class. This finding may suggest that patients after the Fontan procedure can develop an adaptation to chronic CI decrease.

The CMR-derived CI was found to be a predictor of nonelective hospitalizations. A study of the pediatric population in the Mayo Clinic, Rochester, Minnesota, United States, showed that a low CI and elevated pulmonary vascular resistance obtained during cardiac catheterization can identify patients at high risk of Fontan circulation failure. ${ }^{15}$ Our study confirmed that the CMR-derived CI can identify patients at increased risk of Fontan circulation failure.

This study was limited by the small number of patients enrolled. However, SV is a rare disease and the results of this study represent 
the experience of only a single medical center. The second limitation of the study was the lack of the parallel invasive measurement of CI. Despite these limitations, the study demonstrated the prognostic value of a noninvasive parameter that can be obtained by routine diagnostic procedures. Our study also provided an insight into the pathophysiology of Fontan circulation deterioration, since $\mathrm{CI}$ depends on the time from TCPC palliation.

The CMR-derived $\mathrm{CI}$ is not correlated with organ dysfunction parameters. Instead, our evaluation suggested the development of some degree of multiorgan functional tolerance despite chronic organ hypoperfusion. A reduced CMR-derived CI was a predictor of nonelective hospitalizations in the study population.

\section{SUPPLEMENTARY MATERIAL}

Supplementary material is available at www.mp.pl/kardiologiapolska.

\section{ARTICLE INFORMATION}

CONFLICT OF INTEREST None declared.

OPEN ACCESS This is an Open Access article distributed under the terms of the Creative Commons Attribution-NonCommercial-NoDerivatives $4.0 \mathrm{In}$ ternational License (CC BY-NC-ND 4.0), allowing third parties to download articles and share them with others, provided the original work is properly cited, not changed in any way, distributed under the same license, and used for noncommercial purposes only. For commercial use, please contact the journal office at kardiologiapolska@ptkardio.pl.

HOW TO CITE Kowalczyk M, Zieliński P, Marczak M, et al. Cardiac magnetic resonance-derived cardiac index is associated with adverse outcomes in patients with single-ventricle Fontan circulation: a preliminary study. Kardiol Pol. 2021; 79: 336-338. doi:10.33963/KP.15822

\section{REFERENCES}

1 Jacobs ML, Mayer JE Jr. Congenital Heart Surgery Nomenclature and Database Project: single ventricle. Ann Thorac Surg. 2000; 69: 197-204.

2 Mazurak M, Kusa J, Orantek S, Zembala M. The Columbus of cardiovascular surgery. A tribute to Francis Fontan (1929-2018) Kardiol Pol. 2018; 76: 814-815.

3 Smaś-Suska M, Skubera M, Wilkosz T, et al. Noninvasive assessment of liver status in adult patients after the Fontan procedure. Pol Arch Intern Med. 2019; 129: $181-188$.

4 Lee D, Levin A, Kiess M, et al. Chronic kidney damage in the adult Fontan population. Int J Cardiol. 2018; 257: 62-66.

5 Takken T, Tacken MH, Blank AC, et al. Exercise limitation in patients with Fontan circulation: a review. J Cardiovasc Med. 2007; 10: 775-781.

6 Claessen G, La Gerche A, Van De Bruaene A, et al. Heart rate reserve in Fontan patients: chronotropic incompetence or hemodynamic limitation? J Am Heart Assoc. 2019; 9: e012008.

7 Gewillig M. The Fontan circulation. Heart. 2005; 91: 839-846.

8 Hundley WG, Li HF, Hillis LD, et al. Quantitation of cardiac output with velocity-encoded, phase-difference magnetic resonance imaging. Am J Cardiol. 1995; 75: 1250-1255.

9 Carlsson M, Andersson R, Bloch KM, et al. Cardiac output and cardiac index measured with cardiovascular magnetic resonance in healthy subjects, elite athletes and patients with congestive heart failure. J Cardiovasc Magn Reson. 2012; 14: 51.

10 Marino BS, Goldberg DJ, Dorfman AL, et al. Abnormalities in serum biomarkers correlate with lower cardiac index in the Fontan population. Cardiol Young. 2017; 27: 59-68.

11 Gewillig M, Brown SC, Eyskens B, et al. The Fontan circulation: who controls cardiac output? Cardiovasc Thorac Surg. 2010; 10: 428-433.

12 Albakri A. Low-output heart failure: a review of clinical status and meta-analysis of diagnosis and clinical management methods. Clin Med Invest. 2019; 4: 5-11.

13 Mori M, Hebson C, Shioda K, et al. Catheter-measured hemodynamics of adult Fontan circulation: associations with adverse event and end-organ dysfunctions. Congenit Heart Dis. 2016; 11: 589-597.

14 Larsson ES, Eriksson BO, Sixt R. Decreased lung function and exercise capacity in Fontan patients. A long-term follow-up. Scand Cardiovasc J. 2003; 37: 58-63.

15 Egbe AC, Connolly HM, Miranda WR, et al. Hemodynamics of Fontan failure: the role of pulmonary vascular disease. Circ. Heart Fail. 2017; 10: e004515. 